\title{
Density parameter and the anthropic principle
}

\author{
Jaume Garriga \\ IFAE, Departament de Fisica, Universitat Autonoma de Barcelona, 08193 Bellaterra, Barcelona, Spain \\ Takahiro Tanaka \\ Department of Earth and Space Science, Graduate School of Science, Osaka University, Toyonaka 560-0043, Japan
}

\author{
Alexander Vilenkin \\ Institute of Cosmology, Department of Physics and Astronomy, Tufts University, Medford, Massachusetts 02155
}

(Received 25 March 1998; published 25 May 1999)

\begin{abstract}
In the context of open inflation, we calculate the probability distribution for the density parameter $\Omega$. A large class of two field models of open inflation do not lead to infinite open universes, but to an ensemble of inflating islands of finite size, or "quasi-open" universes, where the density parameter takes a range of values. Assuming we are typical observers, the models make definite predictions for the value $\Omega$ we are most likely to observe. When compared with observations, these predictions can be used to constrain the parameters of the models. We also argue that observers should not be surprised to find themselves living at the time when curvature is about to dominate. [S0556-2821(99)04712-8]

PACS number(s): $98.80 . \mathrm{Cq}$
\end{abstract}

\section{INTRODUCTION}

Anthropic considerations have often been used in order to justify the "naturalness" of the values taken by certain constants of nature [1]. In these approaches, it is assumed that the "constants", are really random variables whose range and "a priori" probabilities are determined by the laws of physics. Knowledge of these " a priori" probabilities is certainly useful, but not sufficient to determine the probability for an observer to measure given values of the constants. For instance, some values which are in the "a priori" allowed range may be incompatible with the very existence of observers, and in this case they will never be measured. The relevant question is then how to assign a weight to this selection effect.

A natural framework where these ideas can be applied is inflation. There, the false-vacuum energy of the scalar field which drives the inflationary phase can thermalize in different local minima of its potential, and each local minimum may have a different set of values for the constants of nature. Also, there may be different routes from a false vacuum to a given minimum. In this case all thermalized regions will have the same low energy physics constants, but each route will yield a hot universe with different large scale properties. Here, we shall be concerned with this possibility, where the fundamental constants (such as the gauge couplings or the cosmological constant) are fixed, but other cosmological parameters such as the density parameter or the amplitude of cosmological perturbations are random variables whose distribution is dynamically determined.

In this context, the most reasonable-and predictiveversion of the anthropic principle seems to be the principle of mediocrity [2-6], according to which we are typical observers who shall observe what the vast majority of observers would. Thus, the measure of probability for a given set of constants is simply proportional to the total number of civilizations emerging with those values of the constants. In this paper we shall use this principle in order to calculate the probability distribution for the density parameter $\Omega$.

Standard inflationary models predict $\Omega=1$ with "certainty." What this means is that these models can explain the observed homogeneity and isotropy of the universe only if the universe is flat. However, a class of "open inflation", models which lead to $\Omega<1$ have received some attention in recent years [7-9]. In these models, inflation proceeds in two steps. One starts with a scalar field $\sigma$ trapped in a metastable minimum of its potential $V(\sigma)$. The false vacuum energy drives an initial period of exponential expansion, and decays through quantum nucleation of highly symmetric bubbles of true vacuum. The interior of these bubbles has the geometry of an open Friedmann-Robertson-Walker universe. This accounts for the observed homogeneity and isotropy of the universe. In order to solve the flatness problem a second stage of slow roll inflation inside the bubble is necessary.

In models with a single scalar field $\sigma$, all bubbles have the same value of $\Omega$ which is determined by the number of e-foldings in the second period of inflation. The potential $V(\sigma)$ in such models is assumed to have a rather special form, with a sharp barrier next to a flat slow-roll region, which requires a substantial amount of fine-tuning. Additional tunning is needed to arrange the desired value of $\Omega$. A more natural class of models includes two fields, $\sigma$ and $\phi$, with $\sigma$ doing the tunneling and $\phi$ the slow roll [9]. The simplest example is

$$
V(\sigma, \phi)=V_{t}(\sigma)+\frac{g}{2} \sigma^{2} \phi^{2}
$$

where $V_{0}(\sigma)$ has a metastable false vacuum at $\sigma=0$. After $\sigma$ tunnels to its true minimum $\sigma=v$, the field $\phi$ would drive a second period of slow roll inflation inside the bubble. Depending on the value of $\phi$ at the time of nucleation, the number of $e$-foldings of the second stage of inflation would be different. 
Initially, it was believed [9] that models such as Eq. (1) would yield an ensemble of infinite open universes, one inside each nucleated bubble, and each one with a different value of the density parameter. However, it has been recently realized [10] that this picture is oversimplified. The two field models which allow for variable $\Omega$ do not actually lead to infinite open universes, but to an ensemble of inflating islands of finite size inside of each bubble. These islands are called quasi-open universes. Within each island, the number of $e$-foldings of inflation decreases as we move from the center to the edges. Also, each island is characterized by a different number of $e$-foldings in its central region. As a result, even within the same bubble, different observers will measure a range of values of the density parameter. The picture of the large scale structure of the universe in these models is rather simple, because all bubbles have the same statistical properties. We shall see that the quasiopen nature of inflation is of crucial importance for the calculation of the probability distribution for the density parameter.

In models of quasiopen inflation, such as Eq. (1), $\Omega$ takes different values in different parts of the universe, while the other constants of Nature and cosmological parameters remain fixed. More general models can be constructed where other parameters can change as well, and in Sec. VII we give an example of a model with a variable amplitude of density fluctuations. However, our main focus in this paper is on the models in which only $\Omega$ is allowed to vary.

In order to apply the principle of mediocrity to our models, we will have to compare the number of civilizations in parts of the universe with different values of $\Omega$. Of course, we cannot calculate the number of civilizations. However, since the value of $\Omega$ does not affect the physical processes involved in the evolution of life, this number must be proportional to the number of habitable stars or, as a rough approximation, to the number of galaxies. Hence, we shall set the probability for us to observe a certain value of $\Omega$ to be proportional to the number of galaxies formed in parts of the universe where $\Omega$ takes the specified value.

The principle of mediocrity was applied to calculate the probability distribution for $\Omega$ in an earlier paper [11], which assumed the old picture of homogeneous open universes inside bubbles. A serious difficulty encountered in that calculation was that open universes inside the bubbles have infinite volume and contain an infinite number of galaxies. Thus, to find the relative probability for different values of $\Omega$, one had to compare infinities, which is an inherently ambiguous task. This problem was addressed in [11] by introducing a cutoff and counting only galaxies formed prior to the cutoff. Although the cutoff procedure employed in [11] has some nice properties, it is not unique, and the resulting probability distribution is sensitive to the choice of cutoff [12]. This cutoff dependence, which also appears in other models of eternal inflation $[12,13]$, has led some authors to doubt that a meaningful definition of probabilities in such models is even in principle possible $[12,14]$.

However, this pessimistic conclusion may have been premature. According to the quasiopen picture, $\Omega$ takes all its possible values within each bubble. Since all bubbles are statistically equivalent, it is sufficient to consider a single bubble. Moreover, we can restrict ourselves to a finite (but very large) comoving volume within that bubble, provided that its size is much greater than the characteristic scale of variation of $\Omega$. Thus, we no longer need to compare infinities, and the problem becomes well defined.

The possibility of unambiguous calculation of probabilities in the quasiopen model was our main motivation for revising the analysis of Ref. [11]. Also, we shall give a more careful treatment of the astrophysical aspects of the problem which were discussed rather sketchily in [11].

The paper is organized as follows. In Sec. II we review the main features of quasi-open inflation. In Sec. III we introduce the probability distribution for $\Omega$. A basic ingredient in this distribution will be the anthropic factor $\nu(\Omega)$, which gives the number of civilizations that develop per unit thermalized volume in a region characterized by a certain value of $\Omega$. In Sec. IV we evaluate $\nu(\Omega)$ and calculate the probability distribution for $\Omega$ in the model (1). In Sec. V we extend our results to more general models with arbitrary slow roll potentials for the field $\phi$. In Sec. VI we discuss observational constraints on quasiopen models due to cosmic microwave background (CMB) anisotropies and how these constraints restrict the class of models that give a probability distribution peaked at a non-trivial value of $\Omega$. In Sec. VII we comment on the "cosmic age coincidence," that is, on whether it would be surprising to find ourselves living at the time when the curvature of the universe starts dominating. In Sec. VIII we summarize our conclusions. Some side issues and technical details are discussed in the appendices.

\section{QUASI-OPEN INFLATION}

In this section we shall review the main features of quasiopen models which will be relevant to our discussion. To begin with, we shall consider a model of the form (1). In Sec. $\mathrm{V}$ we shall consider more general slow-roll potentials.

As mentioned in the Introduction, the interior of a bubble is isometric to an open Friedmann-Robertson-Walker universe, with line element

$$
d s^{2}=-d t^{2}+a^{2}(t)\left[d r^{2}+\sinh ^{2} r\left(d \theta^{2}+\sin ^{2} \theta d \varphi^{2}\right)\right] .
$$

The scale factor $a$ obeys the Friedmann equation

$$
H^{2} \equiv\left(\frac{\dot{a}}{a}\right)^{2}=\frac{8 \pi G}{3} \rho+\frac{1}{a^{2}}
$$

At sufficiently early times $(t \rightarrow 0)$, the curvature term in the right-hand side (RHS) dominates over the energy density $\rho$ of the scalar fields, and the scale factor behaves as $a \approx t$.

For the second period of slow roll inflation inside the bubble, the energy density of the scalar fields must be dominated by the potential term

$$
V(\sigma, \phi) \gg \dot{\sigma}^{2}, \dot{\phi}^{2} .
$$

Inside the bubble, the field $\sigma$ quickly settles down to its vacuum expectation value (VEV) $\sigma=v$ with $V_{t}(v)=0$. This 
happens on a time scale of order $t_{0} \sim M^{-1}$, where $M$ is the typical mass scale of $V_{t}$. After that, $\phi$ becomes a free field with constant mass

$$
m^{2}=\frac{g}{2} v^{2}
$$

and the condition for inflation becomes $\phi \gtrsim M_{p}$, where $M_{p}^{2}$ $=G^{-1}$.

An important feature of quasi-open models is the existence of the so-called supercurvature modes for the slow roll field $\phi$. These are modes which are not normalizable on the infinite $t=$ const hyperboloids inside the bubble, but which nevertheless have to be included in the field expansion. The reason is that they are normalizable on the Cauchy surface where equal time commutation relations are imposed. Supercurvature modes are characterized by their eigenvalue of the Laplacian on the 3-hyperboloid. For the model (1), this eigenvalue is given by [10]

$$
\gamma=\frac{1}{8} H_{F}^{2} R_{0}^{4} m^{2} \ll 1 .
$$

Here,

$$
H_{F}^{2}=\frac{8 \pi G}{3} V_{0}(0)
$$

is the Hubble rate during the first stage of false vacuum dominated inflation and $R_{0}$ is the size of the bubble at the time of nucleation, which can be given in terms of the model parameters [15]. Typically, $m^{2} \ll H_{F}^{2}<R_{0}^{-2}$, from where the condition $\gamma \ll 1$ follows.

Around the time $t_{0}$ when $\sigma$ settles down to its VEV, the field $\phi$ will be in a homogeneous and isotropic quantum state with mean squared amplitude given by [10]

$$
f^{2} \equiv\left\langle\phi^{2}\right\rangle \approx\left(\frac{H_{F}}{2 \pi}\right)^{2} \frac{1}{\gamma} .
$$

The presence of the factor $\gamma^{-1}$ reflects the fact that $f$ is dominated by the contribution of supercurvature modes. Introducing $\gamma$ from Eq. (5), we find

$$
f \approx \frac{\sqrt{2}}{\pi} \frac{1}{m R_{0}^{2}} .
$$

Up to numerical factors, this is basically the finite temperature dispersion of a field of mass $m$ at the Rindler temperature given by $T=\left(2 \pi R_{0}\right)^{-1}$. The correlation length of $\phi$ is given by [10] $r \sim \gamma^{-1}$. This means that at the time $t \sim t_{0}$ we can divide the space into regions of co-moving size $r$ $\sim \gamma^{-1} \gg 1$ where the field is coherent. Notice that the size of these regions is much larger than the curvature scale $r=1$.

The parameters of the model can be chosen in such a way that $f$ is close to the Planck scale, and in that case the slow roll field easily reaches inflating values $\phi \sim M_{P}$. For instance, if the potential $V_{t}$ is such that the bubble walls are thick, then $R_{0} \sim M^{-1}$. Taking $M \sim 10^{16} \mathrm{GeV}$ and $m \sim 10^{13}$
$\mathrm{GeV}$, we find $f \sim M_{p}$. In this case, inflating regions of comoving size $r \sim \gamma^{-1}$ where the field is large and positive will be next to inflating regions where the field is large and negative. These two inflating regions will be separated by regions where the field is small and the universe does not inflate.

The parameters can also be such that $f \ll M_{p}$, and in that case most of the regions will not attain an inflating value of $\phi$. Inflation will only happen in those regions where, as a result of a statistical fluctuation, the field happens to be far above its rms value. Since the volume of the hyperboloid is infinite, there will be a small but finite density of these inflating islands inside of each bubble. Those rare "high peaks" will have spherical symmetry. If we take the inflating patch to be centered at $r=0$, the radial profile of the field is given by

$$
\phi\left(t_{0}, r\right) \approx \phi_{0} \frac{\sinh \left[(1-\gamma)^{1 / 2} r\right]}{(1-\gamma)^{1 / 2} \sinh r}
$$

where $\phi_{0} \equiv \phi\left(t_{0}, 0\right)$ is a constant. The probability distribution for $\phi_{0}$ is given by

$$
P\left(\phi_{0}\right) \propto \exp \left[\frac{-\phi_{0}^{2}}{2 f^{2}}\right] .
$$

The variation of $\phi_{0}$ within the bubble results in a positiondependent number of inflationary e-foldings, and thus in a variable density parameter $\Omega$. Note that all other cosmological parameters, such as the amplitude of the density fluctuations, remain fixed throughout the bubble (and are the same for all bubbles). The probability (9) is one of the basic ingredients from which the most probable value of $\Omega$ is calculated.

It should be mentioned that the size of an inflating region can be much larger than the size of the actual "populated" region within it, $r_{p}$, where matter will cluster efficiently into galaxies. The size of the populated region is calculated in Appendix A. This size should be larger than the present horizon, since otherwise we would observe large anisotropies in the galaxy distribution. For $\Omega$ not too close to 1 , the horizon distance is comparable to the curvature scale, $r=1$, and we have to require that $r_{p}>1$. The corresponding constraint on $\gamma$ is obtained in Appendix A.

Equation (9) can be understood from a different perspective, by using the Euclidean approach to the calculation of the nucleation rate. The strategy is to study how this rate is affected by the local value of $\phi$ at the place where the bubble nucleates. This is simple because we only need this Euclidean action to quadratic order. Taking $\phi=0$, we denote by $\sigma_{0}(\tau)$ the $O(4)$ symmetric instanton [16] responsible for vacuum decay. Here, $\tau=i t$ is the Euclidean time, the "radial" coordinate on which the instanton depends. Expanding the Euclidean action $S_{E}$ to second order in perturbations of $\sigma$ and of $\phi$, the perturbations in $\sigma$ and $\phi$ will decouple to quadratic order. Taking $\phi=\phi_{0}=$ const the change in the Euclidean action will simply be

$$
\Delta S_{E}=\int(g / 2) \sigma_{0}^{2}(\tau) \phi_{0}^{2} d^{4} x .
$$


We can approximate the integral by taking $g \sigma_{0}^{2}=m^{2}$ inside the volume of the bubble and $\sigma_{0}=0$ outside. Then we have

$$
\Delta S_{E}=\frac{\pi^{2}}{2} m^{2} R_{0}^{4} \frac{\phi_{0}^{2}}{2} .
$$

From the formula $P \sim \exp \left(-S_{E}\right)$, we essentially recover Eq. (9). Even though we have used the thin wall approximation, we should stress that the coincidence of this "adiabatic" result (where the field $\phi$ is taken as constant) with the field theoretic one (where $\phi$ is quantized in the bubble background and its r.m.s. is evaluated right after bubble nucleation) is also valid for thick walls [10].

As emphasized in [10], the adiabatic approach to the calculation of the distribution of $\phi_{0}$ should be interpreted with caution. It does not mean that the surface $t=t_{0}$ inside the bubble will have a constant value of the field $\phi$. It only gives the probability that a bubble will nucleate with the value of $\phi=\phi_{0}$ near $r=0$. We know, however, that the quantum state of a nucleating bubble is homogeneous, and therefore in the ensemble of bubbles there is nothing special about the point $r=0$. Therefore, this also gives the probability distribution for $\phi$ around any point inside the bubble.

\section{PROBABILITY DISTRIBUTION FOR $\mathbf{\Omega}$}

In this section we shall follow some of the steps used in Ref. [11] for the calculation of the probability distribution for $\Omega$, although the present case will actually be simpler. In the case of Ref. [11], one had to deal with an infinite number of bubbles, each one containing an infinite open universe with a different density parameter. Since the probability for a given set of parameters is roughly proportional to the total volume that ends up having those values of the parameters, one had to face the difficulty of comparing infinite volumes in an eternal inflationary universe [13].

In our case, all bubbles are statistically equivalent. All of them are described by a homogeneous and isotropic quantum state, with $\left\langle\phi^{2}\right\rangle$ given by Eq. (6). Hence, in order to find the probability distribution for $\Omega$ it is sufficient to look at the interior of a single bubble. Also, since the quantum state is homogeneous, we only need to consider the evolution of a patch of finite co-moving size around an arbitrary point on the $t \sim M^{-1}$ hyperboloid. The patch should be sufficiently large that it contains regions with all possible values of $\phi$, distributed according to Eq. (9). Since inflation inside the bubble is not eternal, the number of civilizations resulting from this co-moving patch is finite and there is no need for regularization.

As mentioned in the previous section, at early times the scale factor behaves as $a \approx t$. By the time $t \sim H^{-1}\left(\phi_{0}\right)$, where

$$
H\left(\phi_{0}\right)=(4 \pi G / 3)^{1 / 2} m \phi_{0},
$$

the energy density in the scalar field $\phi$ starts dominating over the curvature term. If the condition for slow roll inflation

$$
\phi_{0}>\phi_{t h} \equiv \frac{M_{p}}{\sqrt{4 \pi}}
$$

is satisfied, then using Eq. (3) the scale factor will subsequently evolve as

$$
a(t) \approx H_{0}^{-1} e^{N(t)},
$$

where $H_{0} \equiv H\left(\phi_{0}\right)$ and

$$
N(t)=\int_{\phi_{0}}^{\phi(t)} \frac{H(\phi)}{\dot{\phi}} d \phi
$$

Using the slow-roll equation of motion for $\phi$

$$
\dot{\phi}=\frac{-m^{2}}{3 H} \phi
$$

we have $N(t) \approx 2 \pi G\left[\phi_{0}^{2}-\phi^{2}(t)\right]$. Since $\phi_{0}$ is actually a slowly varying function of position, the scale factor is a local one, and should be understood as $a\left(t, x^{i}\right)$. Notice that the comoving scale over which $a$ changes is comparable to $\gamma^{-1}$ and hence it is much larger than the curvature scale, so it is meaningful to use the Friedmann equation (3).

The number of $e$-foldings of inflation depends on the local value of $\phi_{0}$ :

$$
a_{t h}\left(\phi_{0}\right) \equiv H_{0}^{-1} e^{N_{t h}\left(\phi_{0}\right)} \approx H_{0}^{-1} e^{2 \pi G\left(\phi_{0}^{2}-\phi_{t h}^{2}\right)},
$$

where $\phi_{t h}$ is defined in Eq. (11). It will be convenient, as a first step, to find the probability distribution for a random "civilization" to live in a region which had a value of the slow roll field equal to $\phi_{0}$ at the beginning of inflation. This is given by

$$
d \mathcal{P}\left(\phi_{0}\right)=P\left(\phi_{0}\right) a_{t h}^{3}\left(\phi_{0}\right) \nu\left(\phi_{0}\right) d \phi_{0} .
$$

Here

$$
P\left(\phi_{0}\right) \propto e^{-\phi_{0}^{2} / 2 f^{2}}
$$

is the probability that a given point on the $t \sim M^{-1}$ hyperboloid will have the value $\phi_{0}$ right after nucleation. Because the number of civilizations is proportional to the volume, we have inserted the total expansion factor during inflation $a_{t h}^{3}\left(\phi_{0}\right)$. Finally, $\nu\left(\phi_{0}\right)$ is the "human factor," which represents the number density of civilizations that will develop per unit thermalized volume as a function of $\phi_{0}$.

Factoring out the dependence on $\nu$,

$$
d \mathcal{P}=\nu\left(\phi_{0}\right) d \widetilde{\mathcal{P}}
$$

the leading exponential behavior of $d \widetilde{\mathcal{P}}$ is

$$
\exp \left(6 \pi G-\frac{1}{2 f^{2}}\right) \phi_{0}^{2}
$$


where we have used Eqs. (12) and (13). The behavior of $\widetilde{\mathcal{P}}$ depends on whether $f$ is large or small compared with $M_{p}$. Defining

$$
\mu \equiv \frac{1}{24 \pi G f^{2}}
$$

it is clear that for $\mu<1 / 2$ large values of $\phi_{0}$ are favored due to the gain in volume factor, and we may expect the universe to be very flat. For $\mu>1 / 2$, the volume factor alone is not sufficient to compensate for the exponential suppression of high peaks. We shall see that the human factor may play an important role in this case.

It is convenient to express the above distribution in terms of the density parameter. Following $[8,11]$, we have

$$
\left[H\left(\phi_{t h}\right) a_{t h}\left(\phi_{0}\right)\right]^{2}=1+B \frac{\Omega}{1-\Omega} \approx B \frac{\Omega}{1-\Omega} .
$$

Here

$$
B \approx \frac{T_{t h}^{2}}{T_{e q} T_{C M B}}
$$

$T_{e q}$ is the temperature at equal matter and radiation density, $T_{t h}$ is the thermalization temperature and $T_{C M B}$ is the temperature of the cosmic microwave background, measured at the same time as $\Omega$. Typically, $B$ is exponentially large, with $(\ln B) \sim 10^{2}$. From Eqs. (13) and (17) we find

$$
d \widetilde{\mathcal{P}}(\Omega)=P\left(\phi_{0}\right) a_{t h}^{3}\left(\frac{d \ln a_{t h}}{d \phi_{0}}\right)^{-1} \frac{d \Omega}{2 \Omega(1-\Omega)} .
$$

Using Eqs. (12), and disregarding the logarithmic dependence on $\Omega$, we find

$$
d \widetilde{\mathcal{P}}(\Omega) \propto \Omega^{1 / 2-3 \mu}(1-\Omega)^{3 \mu-5 / 2} d \Omega,
$$

where $\mu$ is given by Eq. (16). For $\mu>5 / 6$ the probability distribution is peaked at $\Omega=0$, for $\mu<1 / 6$ it is peaked at $\Omega=1$, and for the intermediate range $1 / 6<\mu<5 / 6$ it has two peaks, one at $\Omega=0$ and one at $\Omega=1$. However, it is easily seen that for $\mu>1 / 2$ the highest peak will be at $\Omega=0$ whereas for $\mu<1 / 2$ it will be at $\Omega=1 .{ }^{1}$ Note that all dependence on the particle physics model in Eq. (19) has been compressed into a single parameter $\mu$.

Equation (19) is the same expression that was found in Ref. [11] by considering an ensemble of bubbles with different values of $\Omega$ and using the prescription introduced in [13] for the regularization of infinite volumes. We regard the agreement between the two approaches as a validation of this regularization prescription (in models where regularization is

\footnotetext{
${ }^{1}$ Strictly speaking, the peak would not be exactly at $\Omega=1$ because the Gaussian distribution (14) is only an approximation which ignores the back reaction of the slow roll field on the bubble background. We shall return to this issue in Sec. V.
}

needed). Alternative regularizations proposed in [12] give different results and are therefore disfavored.

Let us now include the human factor $\nu(\Omega)$. As mentioned above, this will play a role for $\mu>1 / 2$, when the expansion alone is not sufficient to compensate for the exponential suppression in $\phi_{0}$ due to tunneling. Since the probability distribution $\widetilde{\mathcal{P}}$ tends to peak near the extremes, it is convenient to work with a logarithmic variable which gives equal measure to each decade in the vicinity of $\Omega=0$ or $\Omega=1$. One such variable is $\ln x$, where

$$
x \equiv \frac{1-\Omega}{\Omega} .
$$

Hence, we shall be interested in the probability density

$$
W(\Omega)=\frac{d \mathcal{P}}{d \ln x} \propto \nu(x) x^{3(\mu-1 / 2)} .
$$

The peak of this distribution will give the most probable value of $\Omega$.

It should be noted that, since the density parameter changes with time, both $\nu(\Omega)$ and $W(\Omega)$ are in principle time dependent. However, this time dependence is somewhat trivial, entering Eq. (21) through the parameter $T_{C M B}$, the temperature at which the density parameter is equal to $\Omega$. What we are actually interested in is the probability distribution for different types of thermalized regions, which is intrinsically time independent. We could, for instance, set $T_{C M B}$ equal to the temperature at recombination, and then the probability distribution would be expressed in terms of $\Omega_{\text {rec }}$, which completely characterizes the history of a given thermalized region. Noting that Friedmann's equation can be rewritten as $x^{-1}=(8 \pi G / 3) \rho a^{2}$, in the matter era we have

$$
x \propto a(t) \propto T_{C M B}^{-1},
$$

where $a$ indicates the scale factor. Hence, in practice, we can use a "gauge invariant"' approach: we shall write $W(\Omega)$ as a function of the product $x T_{C M B}$, which is time independent in the matter era.

\section{THE ANTHROPIC FACTOR $\nu(\Omega)$}

In previous work $[17-19,11,20], \nu(\Omega)$ was taken to be proportional to the fraction of clustered matter $f_{c}$ on a relevant mass scale $M_{g}$. This scale can be chosen as the typical mass of an $L_{*}$ galaxy, $M_{g} \sim 10^{12} M_{\odot}[17,20]$, given that most of the observed luminous matter is in this form. Also, galaxies much smaller than $10^{12} M_{\odot}$ may not be suitable for life, because their gravitational potential would not be able to hold the heavy elements produced in supernovae explosions. Matter will only cluster when the density contrast $\delta\left(M_{g}\right)$ extrapolated from linear perturbation theory exceeds a certain threshold $\delta_{c}$. Hence $f_{c}$ can be estimated as $[21,22]$

$$
f_{c}\left(M_{g}, t\right)=\operatorname{erfc}\left(\frac{\delta_{c}}{\sqrt{2} \sigma}\right)
$$


Here erfc is the complementary error function and $\sigma\left(M_{g}, t\right)$ is the dispersion in the density contrast, also evolved according to linear theory ${ }^{2}[23]$

$$
\sigma\left(M_{g}, t\right)=\frac{5 \sigma_{r e c}\left(M_{g}\right)}{2 x_{r e c}} f(x),
$$

where $x$ is given by Eq. (20) and

$$
f(x)=1+\frac{3}{x}+\frac{3(1+x)^{1 / 2}}{x^{3 / 2}} \ln \left[(1+x)^{1 / 2}-x^{1 / 2}\right] .
$$

The subindex $r e c$ denotes quantities evaluated at the time of recombination. In an open universe, perturbations stop growing after the universe becomes curvature dominated. Since we are interested in the total fraction of clustered matter in the entire history of a given region, we should use in Eq. (23) the asymptotic value of $\sigma$ at large times $(x \rightarrow \infty)$, which approaches a constant.

In a flat universe, the critical density contrast takes the value $\delta_{c} \approx 1.7$. However, it is known that $\delta_{c}$ should be slightly $\Omega$-dependent [24]. The variation is rather small, and $\delta_{c}$ changes by no more than $5 \%$ as $\Omega$ varies from 0.1 to 1 . Here we adopt the value of $\delta_{c}$ estimated in the spherical collapse model as $[25,26]$

$$
\delta_{c}(x)=\frac{3}{2} f(x) g(x),
$$

where

$$
g(x) \equiv 1+\left(\frac{\pi}{x^{1 / 2}(1+x)^{1 / 2}-\sinh ^{-1} x^{1 / 2}}\right)^{2 / 3} .
$$

For $x \rightarrow 0$, we have $\delta_{c}=(3 / 5)(3 \pi / 2)^{2 / 3} \approx 1.69$, as in the case of a flat universe, and for $x \rightarrow \infty$ we have $\delta_{c}=3 / 2$.

Substituting Eqs. (24) and (26) in Eq. (23) and taking the limit $x \rightarrow \infty$ we obtain

$$
\nu=\operatorname{erfc}\left(\frac{3 x_{r e c}}{5 \sqrt{2} \sigma_{r e c}}\right) \equiv \operatorname{erfc}(y) .
$$

The distribution (28) is given as a function of the density parameter at the time of recombination. As mentioned at the end of the last section, in order to compare predictions with observations, it is convenient to express the distribution as a function of $x$ at any temperature $T_{C M B}$. Using Eq. (22) we have

\footnotetext{
${ }^{2}$ This expression for the growth of perturbations is different from the one used in [11]. There, the growth factor from the time of equilibrium of matter and radiation was considered, and a spurious factor of $\Omega$ was included, which was actually due to the uncertainty in the value of the redshift at the time of equilibrium. This factor should actually not be present in the probability distribution for $\Omega$, since the time of equilibrium is the same in all thermalized regions.
}

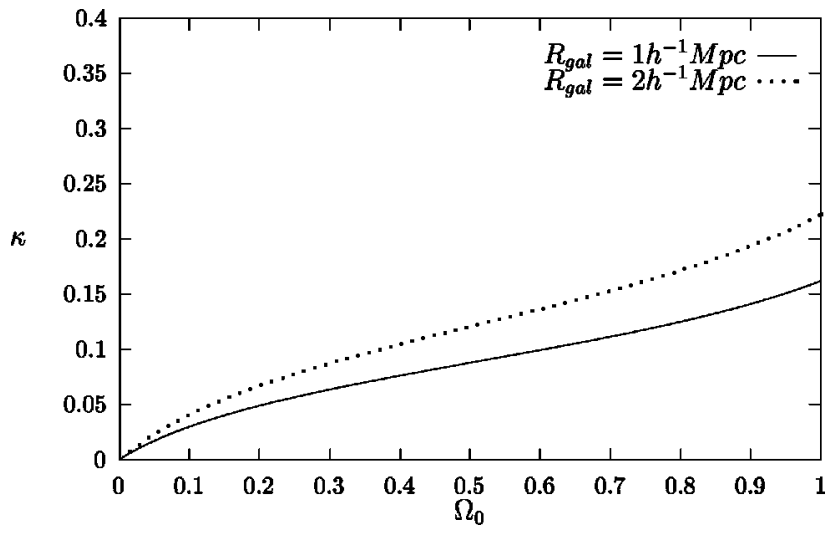

FIG. 1. The coefficient $\kappa$ which relates the variable $y$ to the density parameter $y=\kappa x=\kappa(1-\Omega) / \Omega$, depends on $\sigma_{\text {rec }}$, the value of the density contrast at the time of recombination. Our ability to infer $\sigma_{\text {rec }}$ from CMB observations is limited by the fact that $\Omega_{0}$ in our observable "subuniverse" is not known very precisely. In the figure we plot the inferred value of $\kappa$ for various assumed values of $\Omega_{0}$. The value of $\sigma_{\text {rec }}$ depends moreover on the scale $R_{\text {gal }}$ corresponding to objects of galactic mass. The curve is plotted for two different values of this scale (see Appendix B). The parameter $\kappa$ depends on the temperature at which we observe $\Omega$. Here we have taken $T_{C M B}=2.7 \mathrm{~K}$.

$$
y=\kappa x \equiv \frac{3}{5 \sqrt{2} \sigma_{r e c}} \frac{T_{C M B}}{T_{r e c}} x .
$$

In order to evaluate the coefficient $\kappa$, we need to know $\sigma_{\text {rec }}$. It is clear that $\sigma_{r e c}$ has nearly the same value in all regions where curvature dominates only well after the time of recombination. In principle this value is given in terms of the parameters of our theory of initial conditions.

In practice, we can adjust the parameters of the theory to fit CMB observations. Our ability to infer $\sigma_{r e c}$ from CMB observations is, however, limited by the fact that it depends on the values of $\Omega_{0}$ and $h$ in our visible universe, which are not very well determined. As noted in [20], this limitation also arises in attempts to find the probability distribution for the cosmological constant. Therefore, until determinations of $\sigma_{r e c}$ become more precise, the best one can do is to assume certain values of $\Omega_{0}$ and $h$ and check whether the assumed values fall within the range favored by the resulting probability distribution for $\Omega$. The value of $\kappa$ for $T_{C M B}=2.7 \mathrm{~K}$ is estimated in Appendix B and plotted in Fig. 1 as a function of $\Omega_{0}$. For each value of $\Omega_{0}, h$ has been chosen so that the "shape parameter" $\Gamma \approx \Omega_{0} h \approx .25$ (see Appendix B). Also, there is some uncertainty in the relevant co-moving scale $R_{\text {gal }}$ corresponding to $M_{\text {gal }}$ [20]. In the figure we consider two possibilities, $R_{\text {gal }}=1 \mathrm{~h}^{-1} \mathrm{Mpc}$ and $R_{\text {gal }}$ $=2 \mathrm{~h}^{-1}$ Mpc. For $\Omega_{0}$ in the range $0.1<\Omega_{0}<0.7$ we find that $\kappa \sim 0.1$.

The fraction of clustered matter $\nu$ is shown by a solid curve in Fig. 2 as a function of $y$. In our universe, the density of matter presently clustered in giant galaxies satisfies $\Omega_{\text {gal }}$ $>0.05$ [27], which implies $\nu(y)>0.05 \Omega^{-1}$. The asymptotic value $\nu(y)$ should be even larger. Solving for $y$, we obtain the observational constraint 


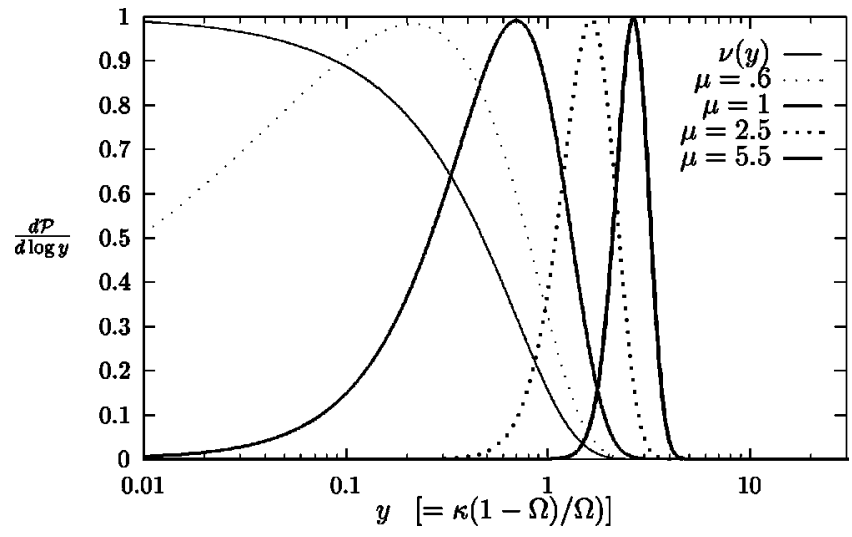

FIG. 2. The probability distribution (31) as a function of $y$, for various values of $\mu$. Also represented is the fraction of clustered matter $\nu(y)$ as a function of $y$.

$$
y<0.9 \text {. }
$$

The distribution

$$
W(\Omega)=\frac{d \mathcal{P}}{d \ln y} \propto \operatorname{erfc}(y) y^{3(\mu-1 / 2)}
$$

gives the probability that a randomly selected civilization is located in a region which had a specified value of $\Omega$ at a given temperature $T_{C M B}$. It is represented in Fig. 2, as a function of $\ln y$, for different values of the parameter $\mu$. The peak value $y_{\text {peak }}$, found from $d W / d y=0$ is plotted in Fig. 3 as a function of $\mu$ (curve $a$ ).

For $y \geq 1$, the error function can be approximated by

$$
\operatorname{erfc}(y) \approx \frac{1}{\sqrt{\pi} y} e^{-y^{2}}
$$

and the peak value can be expressed analytically,

$$
y_{\text {peak }}^{2} \approx \frac{3}{2} \mu-\frac{5}{4} \text {. }
$$

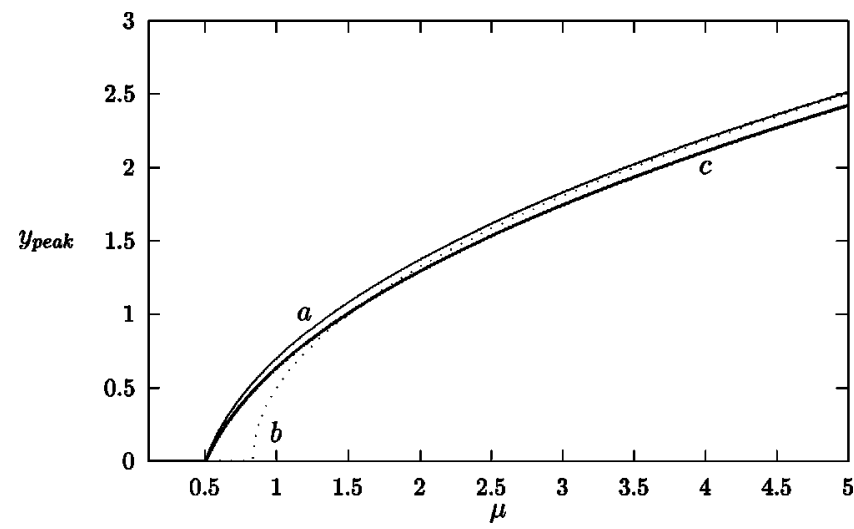

FIG. 3. Peak of the probability distribution (31) (curve $a$ ). The approximate value of $y_{\text {peak }}$ given by Eq. (33) is represented by the curve $b$. Curve $c$ represents the possible effect of helium line cooling failure, as discussed in Appendix C.
This curve is also shown in Fig. 3 (curve $b$ ). Equation (33) can be rewritten as

$$
\left(\frac{1-\Omega}{\Omega}\right)_{\text {peak }}=\kappa^{-1}\left(\frac{3}{2} \mu-\frac{5}{4}\right)^{1 / 2},
$$

which gives the peak value for the density parameter at the temperature $T_{C M B}$. To estimate the width of the distribution (31) we expand $\ln W$ to quadratic order in $\Delta \ln y$ around $y_{\text {peak }}$,

$$
W \approx W_{\text {peak }} \exp \left[-(3 \mu-5 / 2)(\Delta \ln y)^{2}\right] .
$$

Hence, the root mean squared dispersion in $\Omega$ around its peak value will be given by (for $\mu \gtrsim 3 / 2$ )

$$
\Delta \ln \left(\frac{1-\Omega}{\Omega}\right) \sim(6 \mu-5)^{-1 / 2},
$$

while the dispersion in $y$ is independent of $\mu, \Delta y \sim 1 / 2$.

From Fig. 2, we see that as $\mu$ is increased, the probability distribution is sharper and displaced towards larger values of $y$, in agreement with Eqs. (33) and (35). For $\mu=1$, the distribution has a substantial overlap with the region where Eq. (30) is satisfied and the fraction of clustered matter is compatible with observations. For $\mu=5 / 2$ this overlap is smaller, but still non-negligible. However, for $\mu=11 / 2$, the probability density at the point $y \approx 0.9$ is more than two orders of magnitude smaller than at its peak value. Particle physics models which give such high values of $\mu$ are therefore disfavored by observations. As we discussed in Sec. III, the probability distribution is peaked at $\Omega=1$ for $\mu<0.5$. Hence the range of $\mu$ that is of interest to us in this paper is

$$
0.5<\mu \lesssim 3
$$

This corresponds to

$$
0<y_{\text {peak }} \lesssim 2 \text {. }
$$

It should be noted that the peak value for the fraction of clustered matter $\nu\left(y_{\text {peak }}\right)$, depends only on $\mu$, and not on the primordial spectrum of density fluctuations.

So far we assumed that all galactic-size objects collapsing at any time will form luminous galaxies. However, this is not necessarily the case. Galaxies forming at later times have lower density and shallower potential wells. They are vulnerable to losing all their gas due to supernova explosions [28]. Moreover, a collapsing cloud will fragment into stars only if the cooling timescale of the cloud $\tau_{\text {cool }}$ is smaller than the collapse timescale $\tau_{\text {grav }}$, otherwise the cloud would stabilize into a pressure supported configuration [29,28]. The cooling rate of such pressure supported clouds is exceedingly low, and it is possible that star formation in the relevant mass range will be suppressed in these clouds even when they eventually cool. Hence, it is conceivable that galaxies that fail to cool during the initial collapse give a negligible contribution to $\nu(\Omega)$ [28]. The possible effect of cooling failure and related phenomena on the probability distribution for $\Omega$ is discussed in Appendix C, where we show that the effect is 
to shift the peak of the distribution towards larger values of $\Omega$. These effects may be significant, but not dramatic, and Eq. (34) remains valid by order of magnitude. As an illustration, curve $c$ of Fig. 3 shows the peak of the modified distribution when matter which clusters after the time when helium line cooling becomes inefficient is excluded from the anthropic factor $\nu(\Omega)$.

\section{MORE GENERAL MODELS}

In this section, we shall generalize our results to models where the slow roll potential is not necessarily quadratic in $\phi$. In this case, the factor $P\left(\phi_{0}\right)$ in Eq. (13) can be estimated in the adiabatic approximation, where the field $\phi$ is treated as a constant during tunneling, as described at the end of Sec. II. In this approximation we have

$$
P\left(\phi_{0}\right) \propto e^{-S_{E}\left(\phi_{0}\right)}
$$

where $S_{E}\left(\phi_{0}\right)$ is the action of the instanton for bubble nucleation, with the slow roll field frozen to the value $\phi_{0}$.

From Eq. (13), we have

$$
W=\frac{d \mathcal{P}}{d \ln y} \propto \operatorname{erfc}(y) y^{-3 / 2} P\left(\phi_{0}\right) J^{-1},
$$

where, as before

$$
y=\frac{\delta_{c}}{\sqrt{2} \sigma_{r e c}} \frac{2}{5} \frac{T_{C M B}}{T_{r e c}} \frac{1-\Omega}{\Omega},
$$

and we have used Eq. (17) to express the scale factor as a function of $y$. The Jacobian $J$ is given by

$$
J \equiv\left|\frac{d \ln y}{d \phi_{0}}\right|=2 \frac{d \ln a_{t h}}{d \phi_{0}}=-\frac{V^{\prime}}{V}+16 \pi G \frac{V}{V^{\prime}},
$$

where we have used $a_{t h}=H\left(\phi_{0}\right) e^{N}$, and the relation between the hubble rate $H\left(\phi_{0}\right)$ and the slow roll potential $V$ in true vacuum

$$
H_{T}^{2} \equiv H^{2}\left(\phi_{0}\right) \approx \frac{8 \pi G}{3} V\left(\sigma_{T}, \phi_{0}\right)
$$

Here $\sigma_{T}$ is the value of the tunneling field in true vacuum. We have also used the slow roll expression for the number of $e$-foldings

$$
N\left(\phi_{0}\right)=8 \pi G \int \frac{\phi_{0}}{V^{\prime}} d \phi .
$$

Here, as in Eq. (39), $V^{\prime}$ stands for the derivative of $V$ with respect to the slow roll field. Introducing the slow roll parameter

$$
\epsilon \equiv \frac{-\dot{H}_{T}}{H_{T}^{2}} \approx \frac{1}{16 \pi G}\left(\frac{V^{\prime}}{V}\right)^{2} \ll 1
$$

we have

$$
J \approx\left(\frac{16 \pi G}{\epsilon}\right)^{1 / 2}
$$

In many models, the parameter $\epsilon$ hardly changes in the relevant range of $\phi_{0}$, and hence we shall treat it as a small constant parameter.

Extremizing Eq. (38), we find that the peak value of $\phi_{0}$ is given by the condition

$$
\left.\mu\left(\phi_{0}\right)\right|_{\text {peak }}=\frac{1}{2}-\left.\frac{1}{3} \frac{d \ln \operatorname{erfc}(y)}{d \ln y}\right|_{\text {peak }},
$$

where

$$
\mu\left(\phi_{0}\right) \equiv \frac{1}{3} \frac{d S_{E}\left(\phi_{0}\right)}{d \phi} J^{-1} .
$$

Equation (41) is the same condition we found in Sec. IV, and which is plotted in Fig. 3, except that now $\mu$ is a function of $\phi_{0}$, and hence of $y$.

Before we proceed, let us go back to the case discussed in Sec. III of a free slow roll field. Strictly speaking, the expression (14) for $P\left(\phi_{0}\right)$ is just an approximation which is valid only for sufficiently low $\phi_{0}$, when the backreaction of $\phi$ on the bubble background can be neglected. Now we can take this effect into account. For definiteness, let us consider the case where $m \sim 10^{13} \mathrm{GeV}$, and where the tunneling potential $V_{t}(\sigma)$ is such that false and true vacuum are strongly nondegenerate when $\phi=0$. In this case, the radius of the bubble is $R_{0} \sim M^{-1}$ (thick wall bubble), where $M \sim 10^{16} \mathrm{GeV}$ is a typical mass scale in the tunneling potential. Let us denote by $\phi_{d e g}$ the value for which the energy density corresponding to the slow roll potential is equal to the false vacuum energy in the unbroken phase $V_{F} \equiv V_{t}(\sigma=0)$,

$$
\frac{1}{2} m^{2} \phi_{d e g}^{2}=V_{F} \sim \frac{M^{4}}{\lambda},
$$

where $\lambda$ is a self-coupling of the tunneling potential. For $\phi_{0} \ll \phi_{d e g}$, the value of $\mu$ is almost independent of $\phi_{0}$ (this is the situation considered in Sec. III)

$$
\mu\left(\phi_{0} \ll \phi_{d e g}\right) \approx \mu_{0} \equiv \frac{\pi}{48 G} \frac{m^{2}}{M^{4}} .
$$

The masses $M$ and $m$ can be easily adjusted so that $\mu_{0}$ $\ll 1 / 2$. However, for $\phi_{0} \sim \phi_{\text {deg }}$, the Euclidean action $S_{E}\left(\phi_{0}\right)$ increases very steeply with $\phi_{0}$, and so does $\mu$. $^{3}$ In this case, the condition (41) will be satisfied for $\phi_{0} \sim \phi_{\text {deg }}$ $\sim M_{p}\left(\lambda \mu_{0}\right)^{-1 / 2}$, where $M_{p}=G^{-1 / 2}$ is the Planck mass. The corresponding number of $e$-foldings of inflation is given by $N\left(\phi_{0}\right) \approx 2 \pi G \phi_{\text {deg }}^{2} \approx\left(\pi^{2} / 12\right)\left(\lambda \mu_{0}\right)^{-1}$.

Therefore, for $\mu_{0} \ll 1 / 2$, and with a suitable choice of $\lambda$, the peak in the distribution may be adjusted to correspond to

\footnotetext{
${ }^{3}$ Indeed, as $\phi_{0}$ approaches $\phi_{d e g}$, the thin wall approximation starts to apply. Then, from Eqs. (43) and (44) below, we find that the action blows up as we approach degeneracy.
} 
$N \approx(1 / 2) \ln B \approx 60$, where $B$ is defined in Eq. (17). This is compatible with an open universe. However, this case is somewhat trivial, in the sense that the universe can be open only if the maximum allowed value of the slow roll field after tunneling, $\phi_{0}=\phi_{d e g}$, does not drive a long enough period of inflation to make it flat.

Turning to the general case, a more interesting situation arises when $\mu\left(\phi_{0}\right)>1 / 2$ throughout the range of $\phi_{0}$ (see Sec. III). In this case the product of tunneling and volume factors would peak at $\phi_{0}=0$, where the resulting universe would be almost empty, and the anthropic factor $\nu(\Omega)$ is crucial in determining the probability distribution for $\Omega$. For large $\mu$, and using the approximate expression (32) for the error function in Eq. (41) we have

$$
y_{\text {peak }}^{2} \approx \frac{3}{2} \mu\left(\phi_{0}\right)-\frac{5}{4},
$$

which is formally the same expression as Eq. (33).

In the thin wall approximation, we can estimate $\mu$ in terms of $V$ and the bubble radius. For simplicity, we shall also neglect gravitational backreaction. Denoting by $S_{1}$ the tension of the bubble wall, the radius of the bubble at the time of nucleation is given by [16]

$$
R_{0}=\frac{3 S_{1}}{\Delta V\left(\phi_{0}\right)}
$$

where $\Delta V \equiv V_{F}-V\left(\sigma_{T}, \phi_{0}\right)$. Here $V_{F}$ is the potential in false vacuum. For our approximation to be valid, $R_{0}$ should be larger than the thickness of the bubble wall and smaller than the Hubble radius in false vacuum. Under these assumptions, the Euclidean action is given by [16]

$$
S_{E} \approx \frac{\pi^{2}}{2} S_{1} R_{0}^{3}
$$

The derivative of $S_{E}$ can be expressed in terms of the slow roll parameter

$$
\frac{d S_{E}}{d \phi}=3 S_{E} \frac{V^{\prime}}{\Delta V\left(\phi_{0}\right)}=\frac{\pi^{2}}{2} R_{0}^{4} V^{\prime}=\frac{\pi^{2}}{2} R_{0}^{4} V(16 \pi G \epsilon)^{1 / 2},
$$

and finally, from Eq. (41), we have

$$
\mu=\frac{\pi^{2}}{6} R_{0}^{4} V \epsilon
$$

Taking one more derivative of $\ln W$ with respect to $\ln y$, we find

$$
\frac{d^{2} \ln W}{d(\ln y)^{2}} \approx-4 y^{2}-\frac{\pi^{2}}{2} R_{0}^{4} V \epsilon^{2}\left(1+4 \frac{V}{V_{F}-V}\right)
$$

Near $y=y_{\text {peak }}$ we have, setting the first derivative of $W$ to zero and using $\epsilon \ll 1$,

$$
\left.\frac{d^{2} \ln W}{d(\ln y)^{2}}\right|_{\text {peak }} \lesssim-4 y_{\text {peak }}^{2} .
$$

From this we can estimate the dispersion in the distribution of $\Omega$, which is again approximately given by Eq. (35).

\section{CONSTRAINTS FROM CMB ANISOTROPIES}

As we have shown, given a particle physics model which leads to quasi-open inflation, we can predict the probability distribution $P(\Omega)$. Of course, the model also makes predictions for the CMB anisotropies. Comparison of all predictions with observations can be used to constrain the parameters of the particle physics model.

In an open (or quasi-open) universe, CMB anisotropies which are generated during inflation come in three different types. The first type corresponds to scalar fluctuations generated during slow roll inside the bubble, and it affects wavelengths smaller than the curvature scale. These are called subcurvature modes. The corresponding spectrum of temperature fluctuation, characterized by the multipole coefficients $l(l+1) C_{l}$ as a function of $l$, is nearly flat for $l \lesssim 100$. This type of fluctuations is usually believed to give the dominant contribution to the observed plateau in the CMB spectrum.

The second type of anisotropy corresponds to excitations of $\phi$ generated outside the bubble or during the process of tunneling and expansion of the bubble into the false vacuum. These are accounted for by the supercurvature modes discussed in Sec. II (see also [10]). For the models we have considered, the amplitude of temperature anisotropies caused by supercurvature modes is a factor of order $H_{F} / 10 H\left(\phi_{0}\right)$ relative to the subcurvature ones [30]. However, supercurvature modes affect only the very few first multipoles, and hence they cannot explain the observed flat spectrum. For that reason, the constraint $H_{F} \lesssim 10 H\left(\phi_{0}\right)$ is usually imposed.

Finally, there are $\mathrm{CMB}$ anisotropies caused by gravity waves, which can in turn be decomposed into the ones generated during slow-roll and the ones caused by fluctuations of the bubble wall itself [30,31]. Wall fluctuations give the dominant contribution for the few first multipoles, but their contribution decays rapidly with $l$. The waves generated during slow roll give an approximately flat spectrum, whose amplitude is much smaller than that of scalar modes.

The multipole coefficients $C_{l}$ for the temperature anisotropies due to subcurvature modes are given by $[30,32]$

$$
D_{l}^{S} \equiv \frac{l(l+1) C_{l}^{S}}{2 \pi}=\frac{4 \pi G}{25}\left(\frac{H_{T}}{2 \pi}\right)^{2} \frac{1}{\epsilon} b_{l}(\Omega) \quad(l \lesssim 100) .
$$

Here, we have used the notation $H_{T} \equiv H\left(\phi_{0}\right)$ and the slow roll parameter $\epsilon$ given in Eq. (40). The coefficient $b_{l}$ is a slowly varying function of $\Omega$ which can be bounded as 1 $\lesssim b_{l} \lesssim 6$ in the range $1<\Omega<1$.

Supercurvature modes induce temperature anisotropies which for the lowest multipoles can be estimated as $[30,32]$

$$
D_{l}^{S C} \equiv \frac{l(l+1) C_{l}^{S C}}{2 \pi} \sim d_{l}\left(\frac{H_{F}}{H_{T}}\right)^{2} D_{l}^{S}
$$

where $d_{l}(\Omega) \sim 10^{-2}$. 


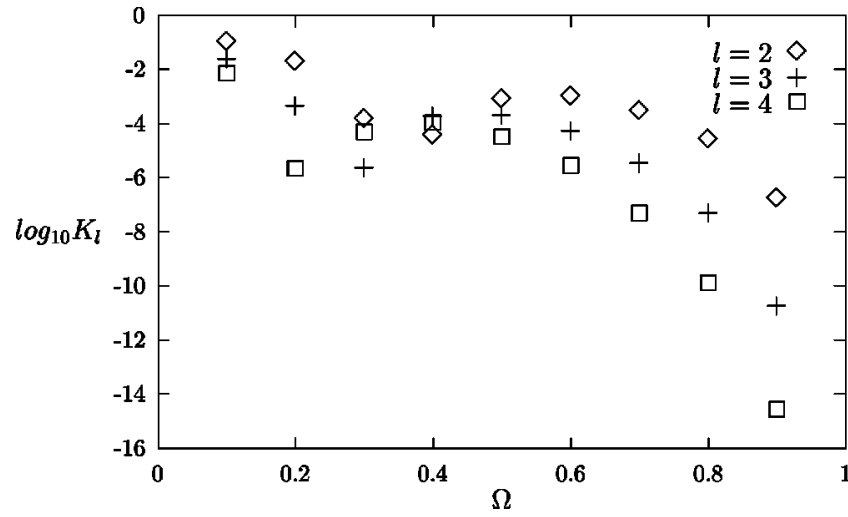

FIG. 4. The coefficient $K_{l}$ for various values of the density parameter.

Let us now consider the contribution to CMB anisotropies from tensor modes. As mentioned above, for the lowest multipoles this is dominated by the domain wall fluctuations $[33,30,32]$. For simplicity, we shall consider the case of a weakly gravitating domain wall, satisfying $G S_{1} R_{0} \ll 1$, where $S_{1}$ is the wall tension. Also, we shall restrict attention to the thin wall case. Then, the anisotropies caused by the wall fluctuations are given by $[32,33,30]$

$$
D_{l}^{W} \approx \frac{2 H_{T}^{2}}{\pi S_{1} R_{0}} c_{l}(\Omega) .
$$

For the first few multipoles, and $\Omega$ in the range .1 to .5 , the coefficient $c_{l}(\Omega)$ is of order $10^{-2}$ [for higher multipoles, $c_{l}$ decays very fast, scaling roughly as $\left.(1-\Omega)^{l}\right]$.

Since $H_{F}^{2}>(8 \pi G / 3) \Delta V$, where $\Delta V$ was introduced in Eq. (43), we have

$$
H_{F}^{2} R_{0}^{2}>24 \pi G \frac{S_{1}^{2}}{\Delta V} \approx 16 G H_{T}^{2}\left(\frac{c_{l}}{D_{l}^{W}}\right) .
$$

From Eq. (45), and using Eqs. (46), (47) and (49), we find

$$
\mu \gtrsim \epsilon^{2} K_{l}\left(\frac{D_{10}^{S}}{D_{l}^{S C}}\right)^{2}\left(\frac{D_{10}^{S}}{D_{l}^{W}}\right)^{2} \frac{1}{D_{10}^{S}},
$$

where the coefficient $K_{l} \equiv 400 \pi^{2} c_{l}^{2} d_{l}^{2} b_{l}^{2} / b_{10}^{3}$ is plotted in Fig. 4 for various values of $l$ and $\Omega$. The inequality (50) turns out to be somewhat restrictive.

In the model given in Eq. (1), the parameter $\epsilon$ $=\left[2 N\left(\phi_{0}\right)\right]^{-1}$ is of order $10^{-2}$. From Fig. 4, the coefficient $K_{l}$ is never smaller than $10^{-4}$ for $\Omega$ in the range .1 to .7 . Hence, we find

$$
\mu \gtrsim 100\left(\frac{D^{S}}{D^{S C}}\right)^{2}\left(\frac{D^{S}}{D^{W}}\right)^{2}\left(\frac{10^{-10}}{D^{S}}\right) .
$$

As discussed in Sec. IV, constraints from the observed fraction of clustered matter imply $\mu \lesssim 3$. On the other hand, observations of $\mathrm{CMB}$ anisotropies require $C_{l}^{i}<10^{-10}$. Hence, we conclude that this model can only satisfy all observa- tional constraints if $\mathrm{CMB}$ anisotropies are not completely dominated by scalar subcurvature modes.

If the observed CMB anisotropies are due to inflation, then we should have $D^{S C}, D^{W} \leqq D^{S} \sim 10^{-10}$, and Eq. (51) gives $\mu \gtrsim 100$. For such values of $\mu$, the peak of the probability distribution is at very low values of $\Omega$, and the corresponding fraction of clustered matter is unacceptably small. It is therefore unlikely that the two-field potential (1) can give a realistic model of open inflation which will explain both a nontrivial value of $\Omega$ and the observed spectrum of CMB fluctuations.

This problem disappears if the observed CMB anisotropies are due to a different source, such as cosmic strings or other topological defects forming at the end of inflation, which would also be responsible for structure formation. Also, the restriction (50) will be less severe if the observed value of $\Omega$ is larger than .7 , since the coefficient $K_{l}$ is then much smaller, or in models with a smaller slow roll parameter $\epsilon \lesssim 10^{-3}$

\section{THE COSMIC AGE COINCIDENCE}

The usual objection against models with $\Omega<1$ is that it is hard to explain why we happen to live at the epoch when the curvature is about to dominate. That is, why

$$
t_{0} \sim t_{c}
$$

where $t_{0}$ is the present time and $t_{c}$ is the time of curvature domination. Observers at $t \ll t_{c}$ would find $\Omega \approx 1$, while observers at $t \gg t_{c}$ would find $\Omega \ll 1$. It appears that one needs to be lucky to live at the time when $\Omega \lesssim 1$. There is another coincidence which is required in open-universe models and which also calls for an explanation. Observationally the epoch of structure formation, when giant galaxies were assembled, is at $z \sim 1-3$, or $t_{G} \sim t_{0} / 3-t_{0} / 8$. On the other hand, the interesting range of $\Omega$ for open universe models is 0.3 $<\Omega<0.9$, which corresponds to $z_{c} \sim 0.1-2$, or $t_{c} \sim 0.3 t_{0}$ $-0.9 t_{0}$. We see that $t_{G}$ and $t_{c}$ are within one order of magnitude of one another. It is not clear why these seemingly unrelated times should be comparable. We could have for example $t_{G} \ll t_{c}$. In this section, we shall argue that the coincidence

$$
t_{G} \sim t_{c} \sim t_{0}
$$

may be not as surprising as it first appears.

Let us begin with the coindidence $t_{G} \sim t_{c}$. In models we are considering here, most of the volume in each quasi-open bubble is occupied by regions with small values of $\Omega$, corresponding to small values of $t_{c}$. Mathematically this is expressed by the fact that the "dehumanized" probability distribution $d \widetilde{\mathcal{P}}(\Omega)$ in Eq. (14) is peaked at $\Omega=0$ (for $\mu$ $>1 / 2)$. On the other hand, the "human factor" $\nu(\Omega)$ suppresses all values of $\Omega$ for which $t_{c}<t_{G}$, so that curvature domination interferes with structure formation. As a result, the peak of the full probability distribution $d \mathcal{P}(\Omega)$ 
$=\nu(\Omega) d \widetilde{\mathcal{P}}(\Omega)$ is shifted to a value of $\Omega$ corresponding to $t_{c} \sim t_{G}$. Hence, we should not be surprised that $t_{c} \sim t_{G}$ in our universe.

It remains to be explained why we live at a time $t_{0}$ $\sim t_{G}$. Clearly, $t_{0}$ could not be much less than $t_{G}$, so we need to explain why we do not have $t_{0} \gg t_{G}$. We now recall Dicke's observation [34] that the time $t_{0}$ is unlikely to be much greater than $t_{G}+t_{\star}$, where $t_{\star} \sim 10^{10} \mathrm{yrs}$ is the lifetime of a typical main sequence star. Noticing that $t_{\star} \sim t_{G}$, we conclude that the expected value of $t_{0}$ is $\sim t_{G}$.

The value of $t_{\star}$ and $t_{G}$ depend only on fundamental constants and on the amplitude of the cosmological density fluctuations. In the models we have considered in this paper, where $\Omega$ is the only variable parameter, these timescales are fixed and one cannot address the question of why they are similar.

If cooling failure (discussed at the end of Sec. IV and in Appendix C) indeed represents a barrier for effective star formation, then it adds yet another timescale which is comparable to the other four we have encountered in this section. This is the time $t_{c f}$ after which collapsing gas clouds of galactic mass cannot fragment and remain pressure supported. This timescale is also determined by fundamental constants, so the coincidence of this scale with $t_{G}$ cannot be explained within our simple model. However, it is easy to generalize the model so that both $\Omega$ and $\sigma_{\text {rec }}$ are variable. For instance, instead of just one slow roll scalar field, we can consider two of them,

$$
V\left(\sigma, \phi_{1}, \phi_{2}\right)=V_{t}(\sigma)+\frac{\sigma^{2}}{2}\left(g_{1} \phi_{1}^{2}+g_{2} \phi_{2}^{2}\right) .
$$

In this case, the two slow roll fields will have different mass inside the bubble. The duration of inflation and the amplitude of density perturbations are determined by the point in the plane $\left(\phi_{1}, \phi_{2}\right)$ where the fields land after tunneling. Changing to polar coordinates on that plane, the number of $e$-foldings of inflation depends basically on the radial coordinate $R$ (how far we are from the bottom of the potential). On the other hand, the amplitude of density perturbations depends on the effective mass along the curve described by the inflaton, which is determined by the angular coordinate $\Theta$.

The volume factor in the probability distribution will be the same on $R=$ const surfaces, whereas the tunneling factor will choose the direction $\Theta$ in which the mass $m^{2}(\Theta)$ $\propto g_{1} \cos ^{2} \Theta+g_{2} \sin ^{2} \Theta$ is the lowest. In our model, $\sigma_{\text {rec }}$ $\propto m(\Theta) N(R) M_{P}^{-1}$, where $N(R) \sim G R^{2}$ is the number of $e$-foldings of inflation and $M_{p}$ is the Planck mass. Low $m$ means large $t_{G}$, because the smaller is $\sigma_{\text {rec }}$, the longer it takes for a perturbation to go nonlinear. Hence, volume and tunneling factors would choose the largest possible $t_{G}$. On the other hand, $t_{G}$ cannot be larger than the cooling boundary $t_{c f}$. Therefore, $t_{G} \sim t_{c f}$ could also be explained in this model. This argument can be regarded as an explanation for the observed amplitude of density fluctuations $Q$ in our universe: the value $Q \sim 10^{-5}$ is selected by the condition $t_{G} \sim t_{c f} \cdot{ }^{4} \mathrm{~A}$ detailed analysis is left for further research.

\section{SUMMARY AND CONCLUSIONS}

We have calculated the probability distribution for the density parameter in models of open inflation with variable $\Omega$. This probability is basically the product of three factors: the "tunneling" factor, which is related to the microphysics of bubble nucleation and subsequent expansion; the volume factor, related to the amount of slow roll inflation undergone in different regions of the universe; and the "anthropic factor,' which determines the number of galaxies that will develop per unit thermalized volume. It is interesting that the expression for the probability (31) depends on the underlying particle physics model through a single dimensionless parameter $\mu$, defined in Eq. (42).

Taking the minimum of the slow roll potential to be at $\phi=0$, the tunneling factor tends to suppress large initial values of $\phi$, favoring low values of $\Omega$. However, only those regions for which $\phi$ is large enough will inflate. Hence, there will be a competition between volume enhancement and "tunneling', suppression.

The most interesting situation occurs when the tunneling suppression dominates over the volume factor. In this case, the product of both would peak at $\Omega=0$, and the anthropic factor $\nu(\Omega)$ becomes essential in determining the probability distribution. In an open universe, cosmological perturbations stop growing when the universe becomes curvature dominated, and for low values of $\Omega$ structure formation is suppressed. The effect of the anthropic factor is, therefore, to shift the peak of the distribution from $\Omega=0$ to a nonzero value of $\Omega$.

As a first approximation $[11,20]$, we have taken $\nu(\Omega)$ to be proportional to the fraction of matter that clusters on the galactic mass scale in the entire history of a certain region. We have found that the peak of the distribution is given by the condition

$$
\kappa\left(\frac{1-\Omega}{\Omega}\right)_{\text {peak }} \approx\left(\frac{3}{2} \mu-\frac{5}{4}\right)^{1 / 2}
$$

where the coefficient $\kappa \sim 10^{-1}$ is defined in Eq. (29). For models with $\mu \sim 1$ (which can be easily constructed), the

\footnotetext{
${ }^{4}$ Anthropic bounds on $Q$ have been previously discussed in Ref. [28]. Tegmark and Rees [28] used the inequality $t_{G}<t_{c f}$ to impose a lower bound on $Q$. To obtain an upper bound, it has been argued $[35,28]$ that for large values of $Q$ galaxies would be too dense and frequent stellar encounters would disrupt planetary orbits. To estimate the rate of encounters, the relative stellar velocity was taken to be the virial velocity $v_{v i r} \sim 200 \mathrm{~km} / \mathrm{s}$, resulting in a bound $Q$ $>10^{-4}$. However, Silk [36] has pointed out that the local velocity dispersion of stars in our galaxy is an order of magnitude smaller than $v_{\text {vir }}$. This gives $Q>10^{-3}$, which is a rather weak constraint. This issue does not arise in the approach outlined in the text above, since in our case large values of $Q$ are suppressed by the tunneling and volume factors in the probability.
} 
probablility distribution for the density parameter $\mathcal{P}(\Omega)$ can peak at values of $\Omega$ such that $x=(1-\Omega) / \Omega \sim 1$ (see Fig. 1). The peaks are not too sharp, with amplitude $\Delta y \approx 1 / 2$, or $\Delta x \approx 5$, so a range of values of $\Omega$ would be measured by typical observers.

The analysis we presented here demonstrates that, given a particle physics model, the probability distribution for $\Omega$ can be unambiguously calculated from first principles. We can also invert this approach and use our results to exclude particle physics models which give the peak of the distribution at unacceptably low values of $\Omega$. This gives the constraint $\mu \lesssim 3$.

An independent constraint on the model parameters can be obtained from $\mathrm{CMB}$ observations. If the observed $\mathrm{CMB}$ anisotropies are to be explained within the same two-field model of open inflation, without adding any extra fields, then we have shown in Sec. IV that the corresponding constraint (if the observed value of $\Omega$ lies in the range .1 to .7) is $\mu$ $\gtrsim 10^{6} \epsilon^{2}$, where $\epsilon$ is the slow roll parameter defined in Eq. (40). Combining both constraints, we obtain a bound on the slow roll parameter

$$
\epsilon \lesssim 10^{-3}
$$

This bound is somewhat restrictive. For instance, for the simple free field model (1), the slow roll parameter is of order $10^{-2}$, and so this model would contradict observations. It is easy, however, to generalize the slow roll potential in order to make $\epsilon$ sufficiently small. If one allows some other source for CMB fluctuations (e.g., topological defects), then the CMB constraint is much less restrictive, and simple models of the form (1) are still viable.

We have advanced anthropic arguments towards explaining the "cosmic age coincidence,"' that is, whether it would be surprising to find that we live at the time when the curvature is about to dominate. We have argued that this is not unexpected. We have also discussed a three-field model in which the amplitude of density fluctuations $Q$ becomes a random variable. We have outlined an argument explaining the observed value $Q \sim 10^{-5}$ in the framework of this model.

While this work was being completed, Hawking and Turok [37], have suggested the possibility of creation of an open universe from nothing (see also [38]). The validity of the instantons describing this process [39], and also their ability to successfully reproduce a sufficiently homogeneous universe, is still a matter of debate and needs further investigation. Clearly, the analysis presented in this paper can be easily adapted to this new framework.

\section{ACKNOWLEDGMENTS}

We would like to thank Andrew Liddle, Martin Rees and Max Tegmark for useful discussions. J.G. thanks the Tufts Cosmology Institute for hospitality. This work has been partially supported by NATO under grant CRG 951301. J.G. acknowledges support from CICYT under contract AEN950882. A.V. acknowledges support from the National Science Foundation.

\section{APPENDIX A: SIZE OF THE POPULATED UNIVERSE AND CLASSICAL ANISOTROPIES}

As mentioned in Sec. II, a quasi-open universe is formed by an ensemble of inflating regions of very large size compared to the curvature scale. Clearly, the central parts of each region will inflate longer and, will have a larger density parameter than the peripheric regions. Hence, the fraction of clustered matter will decrease as we move away from the center. Here we shall estimate the size of the populated region, which, as we shall see, is much smaller than the size of the inflating region.

From Eqs. (21), (17) and (12) we have

$$
\left.d \ln \nu\right|_{\text {peak }}=2 \pi G(6 \mu-3) d \phi_{0}^{2} \sim \frac{\phi_{0}^{2}}{f^{2}} d \ln \phi_{0} .
$$

This equation gives the variation of $\nu$ due to the gradients in $\phi$ as we move away from a typical civilization which measures the peak value of $\Omega$. [The estimate in Eq. (A1) holds provided that $\mu$ is not too close to $1 / 2$, say $\mu \geqslant$.6.]

Taking this civilization to be located at $r=0$, the gradients can be decomposed in multipoles. For $l=0, d \phi_{0}$ can be found from Eq. (8). For $r \sim 1$ (which for low $\Omega$ roughly corresponds to the present Hubble distance) we have $d \ln \phi_{0} \sim \gamma$. Combining with Eq. (A1) we find that $\nu$ changes by

$$
\delta \ln \nu \sim \frac{\gamma\left(\phi_{0}^{\text {peak }}\right)^{2}}{f^{2}} \equiv X
$$

over the Hubble distance.

For $X \ll 1, \nu$ would not change appreciably on cosmological scales. Using Eq. (8), the co-moving size of the populated universe can be estimated as the distance at which $\nu$ drops by an order of magnitude,

$$
r_{p} \sim X^{-1}
$$

For $\mu>1 / 2$ we need $\phi_{0}^{2} \gg f^{2}$ in order to have sufficiently long inflation. Hence we find that the size of the populated region is larger than the curvature scale but still much smaller than the size of the quasi-open island, $1 \ll r_{p} \ll \gamma^{-1}$.

For $X \gg 1$ we can use Eq. (8) for small $r$ to obtain

$$
d \ln \phi_{0} \approx \gamma \frac{r^{2}}{6} \text {. }
$$

In that case, the size of the populated universe can be estimated as

$$
r_{p} \sim X^{-1 / 2} \ll 1
$$

and the human factor would drop by several orders of magnitude within our Hubble radius. Clearly, we should not expect to lie precisely at the center of the hospitable region, but rather at the outskirts, and then we would observe a large anisotropy in $\nu$ around us. This can be confirmed by analyzing the $l>0$ supercurvature modes. The amplitude of $l>0$ modes is of order [10] 


$$
\delta \phi_{0} \sim \gamma^{1 / 2} f r^{l}
$$

Combining with Eq. (A1) we have

$$
\delta \ln \nu \sim X^{1 / 2} r^{l}
$$

For large $X$, the $l=1$ anisotropy in $\nu$ becomes of order one at the distance $r_{p}$, as expected.

If $\nu$ is proportional to the fraction of clustered matter, as we have assumed in the preceding section, a large drop in this quantity is already excluded by observations [40], so the constraint

$$
X \equiv \frac{\gamma\left(\phi_{0}^{\text {peak }}\right)^{2}}{f^{2}} \lesssim 1
$$

must be imposed on our model.

This constraint is relevant to the question of classical anisotropies in a quasi-open universe, discussed in Ref. [41]. To an observer living at large distances from the center of the island $r \gg 1$ the universe would look anisotropic, with $d \phi_{0} \sim \gamma \phi_{0}$ over the curvature scale around that point. For $X>1$ this anisotropy would be larger than the $l>0$ quantum fluctuations from supercurvature modes (A5). However, as shown above, for $X>1$ the typical observer must be at a distance $r \sim X^{-1} \lesssim 1$ from the center of the island, and the arguments of Ref. [41] do not apply. Hence, even though the constraint (A6) coincides with the one derived in [41] (where a single island was considered and the universe was not taken to be homogeneous on very large scales), its interpretation is very different. It does not arise from requiring that the classical CMB anisotropy should be smaller than the $l$ $>1$ supercurvature anisotropy but from demanding that the factor $\nu$ determining the density of civilizations should be isotropic around us.

On the other hand, for the simple model (1), one can find a much stronger constraint on $X$ by combining the bounds from the observed isotropy of the CMB discussed in Sec. VI, with the bounds on the observed fraction of clustered matter. Indeed, the supercurvature anisotropy can be expressed as

$$
D_{l}^{S C} \sim 10^{-6} \mu^{-2} X
$$

where $X$ was defined in Eq. (A2). Using the constraints from the observed fraction of clustered matter $\mu \lesssim 3$ [see Eq. (36)] and requiring $D_{l}^{S C} \lesssim 10^{-10}$, this results in

$$
X \lesssim 10^{-4} \mu^{2} \lesssim 10^{-3},
$$

a much stronger constraint than Eq. (A6). Hence, the size of the populated universe should be at least $10^{3}$ times larger than the curvature scale in this model.

\section{APPENDIX B: EVALUATION OF $\kappa$}

As mentioned at the end of Sec. VI, in order to predict the expected values of $\Omega$ at $T_{C M B}=2.7 \mathrm{~K}$ in our part of the universe, we need to know $\mu$, as well as the coefficient

$$
\kappa=\frac{3}{5 \sqrt{2} \sigma_{r e c}} \frac{T_{C M B}}{T_{r e c}}
$$

that relates $x$ to $y$. For the temperatures we take $T_{C M B} \approx 2.7 \mathrm{~K}$ and $T_{r e c}=1100 T_{C M B}$. The main unknown in this coefficient is $\sigma_{\text {rec }}$.

The value of $\sigma_{\text {rec }}$ is, to a very good approximation, the same in all thermalized regions. Hence it can be inferred from measurements of $\mathrm{CMB}$ anisotropies on large angular scales in our observable region. Since we are interested in relatively small scales, we also need to make some assumptions about the power spectrum of density fluctuations. We shall take a scale invariant cold dark matter (CDM) adiabatic spectrum. As we shall see, our ability to infer the precise value of $\sigma_{\text {rec }}$ will be limited by the fact that the density parameter $\Omega_{0}$ in the observable part of our universe is not known very precisely. Hence, we shall leave it as a free parameter. We emphasize that $\Omega_{0}$ is the value of the density which is actually realized in our universe today, and whose precise value we do not know yet. This should not be confused with the random variable $\Omega$ which appears in the probability distributions, and which takes different values in different regions.

In order to determine $\sigma_{r e c}$, we note that

$$
\sigma_{r e c}(R)=A^{-1}\left(\Omega_{0}\right) \sigma_{0}(R),
$$

where $\sigma_{0}(R)$ is the present density contrast on the relevant scale $R$ and $A\left(\Omega_{0}\right)$ is the factor by which linear perturbations have grown from the time of recombination until the present time. In an open universe, this factor is given by [23]

$$
A\left(\Omega_{0}\right)=\frac{5}{2} \frac{f\left(x_{0}\right)}{x_{r e c}},
$$

where $x_{0}$ and $x_{r e c}$ are the values of $(1-\Omega) / \Omega$ in our observable universe at present and at recombination respectively. The function $f$ is given in Eq. (25).

With this, we have

$$
\kappa=\frac{3}{2 \sqrt{2} \sigma_{0}} \frac{f\left(x_{0}\right)}{x_{0}},
$$

where we have used the fact that $x T=$ const in the matter era.

The present linear density contrast $\sigma_{0}$ is given by $[22,20]$

$$
\sigma_{0}\left(R_{\text {gal }}\right)=\left(c_{100} \Gamma\right)^{2} \delta_{H} K^{1 / 2}\left(R_{\text {gal }}\right) .
$$

Here $c_{100} \approx 2997.9$ is the speed of light in units of $100 \mathrm{~km} \mathrm{~s}^{-1}, \delta_{H}$ is the dimensionless amplitude at horizon crossing (which can be inferred from COBE measurements), $\Gamma=\Omega_{0} h$ is the "shape parameter,' with $h$ the present hubble rate in units of $100 \mathrm{~km} \mathrm{~s}^{-1} \mathrm{Mpc}^{-1}$ (we ignore the effect of baryon density in this expression for $\Gamma$ ), and $K$ contains the information on the power spectrum and the length scale $R_{g a l}$ we are considering.

For a scale invariant spectrum, $K$ is given by [20] 


$$
K(R) \equiv \int_{0}^{\infty} q^{3} T^{2}(q) W^{2}\left(q R h \Gamma \quad \mathrm{Mpc}^{-1}\right) d q
$$

where the transfer function $T$ in the CDM model can be approximated as [42]

$$
\begin{aligned}
T(q)= & \frac{\ln (1+2.34 q)}{2.34 q}\left[1+3.98 q+(16.1 q)^{2}\right. \\
& \left.+(5.46 q)^{3}+(6.71 q)^{4}\right]^{-1 / 4},
\end{aligned}
$$

and the top-hat window function $W$ in momentum space is given by

$$
W(u)=\frac{3}{u^{3}}(\sin u-u \cos u) .
$$

In order to find numerical estimates, we shall consider [20] $R_{\text {gal }}=1-2 \mathrm{~h}^{-1} \mathrm{Mpc}$. Roughly speaking, this corresponds to the scale whose baryon content collapses to form a galaxy with a mass comparable to that of the Milky Way. Also, by requiring that CDM predictions correctly reproduce the statistics for galaxy distribution on scales of tens of megaparsecs [43], the shape parameter is constrained to be in the range

$$
\Gamma \approx 0.25 \pm 0.05
$$

For our estimates, we shall take $\Gamma=.25$. With this, we find

$$
K\left(1 \mathrm{~h}^{-1} \mathrm{Mpc}\right) \approx 0.049, \quad K\left(2 \mathrm{~h}^{-1} \mathrm{Mpc}\right) \approx 0.026
$$

For the dimensionless amplitude $\delta_{H}$ we shall use the fitting function given by Liddle et al. [22]

$$
\delta_{H}(\Omega)=\left(4.10+8.83 \Omega-8.50 \Omega^{2}\right)^{1 / 2} \times 10^{-5} .
$$

Hence, the coefficient

$$
\kappa=\frac{3}{2 \sqrt{2}\left(c_{100} \Gamma\right)^{2} K^{1 / 2}} \frac{f\left(x_{0}\right)}{\delta_{H}\left(\Omega_{0}\right) x_{0}}
$$

will be sensitive to our ignorance of the value of $\Omega_{0}$ in our universe, as mentioned above.

In Fig. 1 we plot $\kappa$ as a function of $\Omega_{0}$ for the two chosen values of the scale $R_{g a l}$.

\section{APPENDIX C: EFFECTS OF COOLING FAILURE}

As mentioned at the end of Sec. IV, fragmentation of gas clouds will only occur if the cooling timescale $\tau_{\text {cool }}$ is smaller than the timescale needed for gravitational collapse $\tau_{\text {grav }}$. Because of this, fragmentation will be suppressed after a certain critical time $t_{*}$. Here we shall investigate the possibility [28] that clouds collapsing at $t>t_{*}$ do not effectively form stars even after they eventually cool. We shall see that, as a consequence, the peak of the distribution will be shifted to somewhat larger values of $\Omega$.

The density of the virialized collapsing cloud $\rho_{v i r}$ is given by $[28,25]$

$$
\rho_{v i r} \sim 10^{2}\left(G t_{v i r}^{2}\right)^{-1}
$$

where $t_{v i r}$ is the time at which the collapse occurs. The virialization temperature can be estimated as $T_{v i r} \sim m_{p} v_{v i r}^{2}$ $\sim m_{p}\left(G^{3} \rho_{v i r} M_{g}^{2}\right)^{1 / 3}$. Here $m_{p}$ is the proton mass, and $v_{v i r}$ is the virial velocity $v_{v i r} \sim\left(G M_{g} / L\right)^{1 / 2}$, where $L$ is the size of the collapsed object. The later an object collapses, the colder and rarer it will be.

The cooling rate $\tau_{\text {cool }}^{-1}$ of a gas cloud of fixed mass depends only on its density and temperature, but as shown above both of these quantities are determined by $t_{v i r} .{ }^{5}$ The timescale needed for gravitational collapse is $\tau_{\text {grav }} \sim t_{v i r}$. Therefore, the condition $\tau_{\text {cool }}<\tau_{\text {grav }}$ gives an upper bound $t_{*}$ on the time at which collapse occurs. Matter that clusters after that time should not contribute to the anthropic factor $\nu(\Omega)$.

Various cooling processes such as bremsstrahlung and line cooling in neutral hydrogen and helium were considered in Ref. [28]. For a cloud of mass $M_{g} \approx 10^{12} M_{\odot}$, cooling turns out to be efficient ${ }^{6}$ for

$$
t<t_{*} \approx 3 \times 10^{10} \mathrm{yr}
$$

This value of $t_{*}$ should be taken only as indicative, since the present status of the theory does not allow for very precise estimates.

From the time of recombination to the time $t_{*}$ fluctuations will grow by the factor [23]

$$
G_{*}(\Omega)=\frac{5}{2 x_{r e c}} f\left(x_{*}\right),
$$

whereas the critical density contrast is given by

$$
\delta_{c}=\frac{3}{2} f\left(x_{*}\right) g\left(x_{*}\right) \text {, }
$$

where $f(x)$ and $g(x)$ are given in Eqs. (25) and (27). Following the steps that lead to Eq. (28) we now find

$$
\nu=\operatorname{erfc}\left[\kappa x g\left(x_{*}\right)\right]
$$

Noting that in the matter era [23]

$$
t=t_{\text {rec }} \frac{x^{1 / 2}(1+x)^{1 / 2}-\sinh ^{-1} x^{1 / 2}}{x_{r e c}^{1 / 2}\left(1+x_{r e c}\right)^{1 / 2}-\sinh ^{-1} x_{r e c}^{1 / 2}}
$$

and using $x_{r e c} \ll 1$ we have

\footnotetext{
${ }^{5}$ Actually, the fraction of baryonic matter $X_{b}$ is also relevant for cooling. Following [28] we shall take $X_{b} \sim 0.1$.

${ }^{6}$ This upper bound on $t$ is determined by line cooling in helium. For $M_{g} \approx 10^{12} M_{\odot}$ there is also a narrow range of time near $t \approx 3 \times 10^{11} \mathrm{yr}$ where cooling is again efficient due to hydrogen line cooling. However, the range is very narrow and we shall disregard the galaxies which may form during this short late period.
} 


$$
g\left(x_{*}\right) \approx 1+\left(\frac{3 \pi t_{r e c}}{2 t_{*}}\right)^{2 / 3} \frac{1}{x_{r e c}}=1+\frac{\Delta_{*}}{x}
$$

where

$$
\Delta_{*}=\frac{T_{r e c}}{T_{C M B}}\left(\frac{3 \pi t_{r e c}}{2 t_{*}}\right)^{2 / 3} .
$$

Therefore, the fraction of matter that clusters on a given scale before the critical time is basically obtained by shifting $y$ in Eq. (28) by the constant $\kappa \Delta_{*}$

$$
\nu=\operatorname{erfc}\left(y+\kappa \Delta_{*}\right) \text {. }
$$

Using the values $\Omega_{0}=.5$ and $h=.5$ for our observable universe in order to infer $\kappa$ (see Fig. 1) and $t_{\text {rec }}$ $\approx 5.6 \times 10^{12}\left(\Omega_{0} h^{2}\right)^{-1 / 2} \mathrm{~s}$, we have $\kappa \Delta_{*} \approx 0.2$ (as in Appendix B, we have used $\left.T_{r e c}=1100 T_{C M B}\right)$. The peak of the modified probability distribution is plotted in Fig. 3 (curve $c$ ) as a function of $\mu$, next to the original curve $a$ where cooling failure is neglected. Asymptotically both curves differ only by $\Delta y_{\text {peak }} \approx \kappa \Delta_{*} / 2=0.1$. This is much smaller than the width of the distribution $\Delta y \sim 0.5$, so the effect is rather small. In Fig. 5(a) we plot the probability distribution as a function of $x=(1-\Omega) / \Omega$ (at the temperature $T_{C M B}=2.7 \mathrm{~K}$ ) for three different values of $\mu$, without taking into account cooling effects. For comparison, in Fig. 5(b) we show the modified distribution when matter that clumps after time $t_{*}$ is disregarded.

We note that even if cooling is efficient, the density of the protogalactic cloud is likely to affect the number and the mass distribution of stars in the resulting galaxy. Masses of suitable stars should be large enough to provide the necessary luminosity and small enough so that the stellar lifetime is sufficient to evolve intelligent life. It is conceivable that the number of such stars drops with the density, in which case the upper bound on $t$ should be stronger than Eq. (C1). Again, galaxy formation is not understood to the extent that would allow us to estimate this upper bound with accuracy. However, since we do not observe many giant galaxies form-

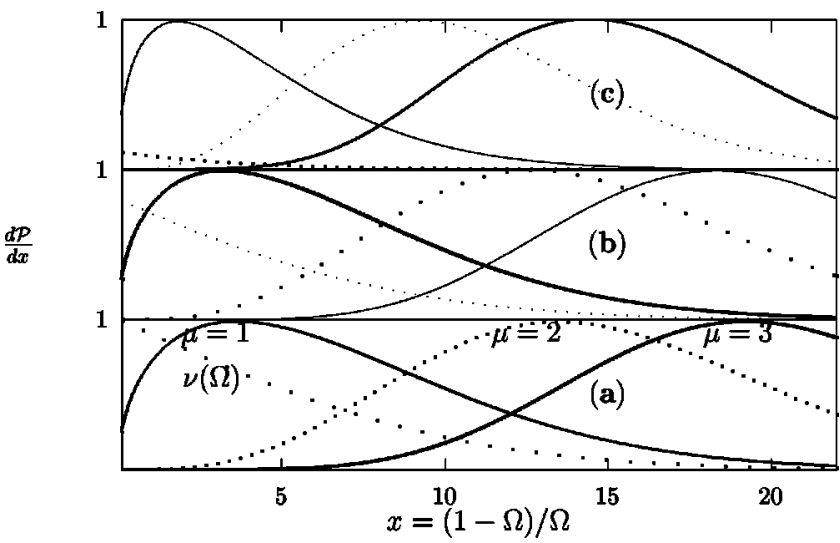

FIG. 5. The probability distribution for $\Omega$ is sensitive to the fact that objects which collapse at very late times have very low density, and therefore may be unsuitable for life. Neglecting these "selection" effects, frame (a) shows the probability distribution for $\Omega$ for various values of $\mu$ (the value of $\Omega$ is the one measured at the temperature $\left.T_{C M B}=2.7 \mathrm{~K}\right)$. In this case, the anthropic factor $\nu(\Omega)$ (also shown in the plot) is just proportional to the total fraction of matter that clusters on the galactic mass scale in the entire history of a particular region. In frame (b) we disregard matter which clusters after the time when helium line cooling becomes inefficient, so that the collapsed galactic mass objects cannot fragment into stars. Finally, as a more extreme case, in frame (c) we disregard matter that clumps after the time $t_{*} \approx 3 \times 10^{9} \mathrm{yr}$, since we do not see many giant galaxies forming at redshifts lower than $z=2$.

ing at redshifts lower than $z=2$, we may consider as a third possibility the case where matter that clumps after the time $t_{*} \approx 3 \times 10^{9} \mathrm{yr}$ is excluded from the anthropic factor. This corresponds to $\kappa \Delta_{*} \approx 1$. Even in this extreme case the shift in the peak $\left|\Delta y_{\text {peak }}\right| \leqslant \kappa \Delta_{*} / 2 \approx 0.5$ is of the same order of magnitude as the width of the distribution $\Delta y \sim 0.5$ [see Eq. (35)]. The new distribution as a function of $x$ is plotted in Fig. 5(c).

Therefore, we find that the impact of these effects on the probability distribution may be significant, but not dramatic, and Eq. (34) is still valid by order of magnitude.
[1] B. Carter, in I.A.U. Symposium, edited by M. S. Longair (Reidel, Dordrecht, 1974), Vol. 63; B. B. Carr and M. J. Rees, Nature (London) 278, 605 (1979); J. Barrow and F. Tipler, The Anthropic Cosmological Principle (Clarendon, Oxford, England, 1986); S. Weinberg, Phys. Rev. Lett. 59, 2607 (1987); A. D. Linde, Particle Physics and Inflationary Cosmology (Harwood Academic, Chur, Switzerland, 1990); J. GarciaBellido and A. Linde, Phys. Rev. D 51, 429 (1995).

[2] A. Vilenkin, Phys. Rev. Lett. 74, 846 (1995).

[3] A very similar approach was used by Carter [4], Leslie [5] and Gott [6] to estimate the expected lifetime of our species. Gott also applied it to estimate the lifetimes of various political and economic structures, including the journal "Nature" where his article was published.

[4] B. Carter (unpublished).
[5] J. Leslie, Mind 101, 521 (1992).

[6] J. R. Gott III, Nature (London) 363, 315 (1993).

[7] J. R. Gott, Nature (London) 295, 304 (1982); J. R. Gott and T. S. Stalter, Phys. Lett. 136B, 157 (1984); A. Guth and E. Weinberg, Nucl. Phys. B212, 321 (1983); M. Sasaki, T. Tanaka, K. Yamamoto, and J. Yokoyama, Phys. Lett. B 317, 510 (1993); K. Yamamoto, M. Sasaki, and T. Tanka, Astrophys. J. 455, 412 (1995); M. Bucher, A. Goldhaber, and N. Turok, Phys. Rev. D 52, 5538 (1995); A. M. Green and A. R. Liddle, ibid. 55, 609 (1997); J. Garcia-Bellido and A. Linde, ibid. 55, 7480 (1997).

[8] M. Bucher, A. S. Goldhaber, and N. Turok, Phys. Rev. D 52, 3314 (1995).

[9] A. D. Linde, Phys. Lett. B 351, 99 (1995); A. D. Linde and A. Mezhlumian, Phys. Rev. D 52, 6789 (1995). 
[10] J. Garcia-Bellido, J. Garriga, and X. Montes, Phys. Rev. D 57, 4669 (1998).

[11] A. Vilenkin and S. Winitzki, Phys. Rev. D 55, 548 (1997).

[12] A. Linde, D. Linde, and A. Mezhlumian, Phys. Rev. D 49, 1783 (1994); A. Linde and A. Mezhlumian, ibid. 53, 4267 (1996).

[13] A. Vilenkin, Phys. Rev. D 52, 3365 (1995).

[14] J. Garcia-Bellido and A.D. Linde, Phys. Rev. D 51, 429 (1995).

[15] S. Parke, Phys. Lett. 121B, 313 (1983).

[16] S. Coleman, Phys. Rev. D 15, 2929 (1977); S. Coleman and F. De Luccia, ibid. 21, 3305 (1980).

[17] G. Efstathiou, Mon. Not. R. Astron. Soc. 274, L73 (1995).

[18] A. Vilenkin, in Cosmological Constant and Evolution of the Universe, edited by K. Sato, T. Suginohara, and N. Sugiyama (Universal Academy Press, Tokyo, 1996).

[19] S. Weinberg, in Critical Dialogues in Cosmology, edited by N. Turok (World Scientific, Singapore, 1997).

[20] H. Martel, P. R. Shapiro, and S. Weinberg, Astrophys. J. 492, 29 (1998).

[21] W. H. Press and P. Schechter, Astrophys. J. 187, 425 (1974).

[22] A. R. Liddle, D. H. Lyth, D. Roberts, and P. P. T. Viana, Mon. Not. R. Astron. Soc. 278, 644 (1996).

[23] P. J. E. Peebles, The Large Scale Structure of the Universe (Princeton University Press, Princeton, NJ, 1980).

[24] P. B. Lilje, Astrophys. J. Lett. 386, L33 (1992); S. Colafrancesco and N. Vittorio, Astrophys. J. 422, 443 (1994).

[25] C. Lacey and S. Cole, Mon. Not. R. Astron. Soc. 262, 627 (1993).

[26] A careful treatment of the variation of $\delta_{c}$ in the case of a flat universe with a cosmological constant has been given by Martel, Shapiro and Weinberg (MSW) who calculated the fraction of clustered matter $f_{c}$ to find an anthropic probability distribution for $\Lambda$ [20]. They considered density fluctuations having the form of a spherical overdense region surrounded by a compensating spherical underdense shell, with a fixed ratio $s$ of overdense to underdense volumes, and calculated $f_{c}$ using the exact collapse criterion for such fluctuations. MSW emphasize that their results are insensitive to the choice of $s$. Now, we note that for large $s$, the formula they obtained for $f_{c}$, their Eq. (26), reduces to the Press-Schechter formula (23) that we use here, with $\delta_{c} \approx 1.63$. This indicates that Press-Schechter formalism would be an adequate approximation in MSW problem as well.

[27] P. J. E. Peebles, Principles of Physical Cosmology (Princeton University Press, Princeton, NJ, 1993).

[28] M. Tegmark and M. Rees, Astrophys. J. 499, 526 (1998).

[29] M. J. Rees and J. P. Ostriker, Mon. Not. R. Astron. Soc. 179, 541 (1977); J. Silk, Astrophys. J. 211, 638 (1977).

[30] M. Sasaki and T. Tanaka, Phys. Rev. D 54, R4705 (1996); K. Yamamoto, M. Sasaki, and T. Tanaka, ibid. 54, 5031 (1996); M. Sasaki, T. Tanaka, and Y. Yakushige, ibid. 56, 616 (1997).

[31] J. Garriga, X. Montes, M. Sasaki, and T. Tanaka, Nucl. Phys. B513, 343 (1998).

[32] J. Garcia-Bellido, Phys. Rev. D 54, 2473 (1996); ibid. 56, 3225 (1997); "Single Bubble Open Inflation: An Overview,' CERN report, hep-ph/9803270.

[33] J. Garriga, Phys. Rev. D 54, 4764 (1996).

[34] R. H. Dicke, Nature (London) 192, 440 (1962).

[35] A. Vilenkin, talk given at the first RESCEU Symposium, Tokyo (1995).

[36] J. Silk (private communication).

[37] S. W. Hawking and N. Turok, Phys. Lett. B 425, 25 (1998).

[38] A. D. Linde, Phys. Rev. D 58, 083514 (1998); W. Unruh, U.B.C. report, gr-qc/9803050; R. Bousso and A. Linde, Phys. Rev. D 58, 083503 (1998); Z. C. Wu, Gen. Relativ. Gravit. 30, 1639 (1998).

[39] A. Vilenkin, Phys. Rev. D 57, 7069 (1998).

[40] See, e.g., M. Davis, in Critical Dialogues in Cosmology, edited by N. Turok (World Scientific, Singapore, 1997), for evidence of homogeneity of large-scale galaxy distribution.

[41] J. Garriga and V. F. Mukhanov, Phys. Rev. D 56, 2439 (1997).

[42] J. M. Bardeen, J. R. Bond, N. Kaiser, and A. S. Szalay, Astrophys. J. 304, 15 (1986).

[43] J. A. Peacock and S. J. Dodds, Mon. Not. R. Astron. Soc. 267, 1020 (1994). 